\title{
Long noncoding RNA Lnc-EGFR promotes cell proliferation and inhibits cell apoptosis via regulating the expression of EGFR in human tongue cancer
}

\author{
TINGTING SUN, LIN TANG and MIAOMIAO ZHANG \\ Department of Orthodontics, The First Affiliated Hospital of Harbin Medical \\ University, Harbin, Heilongjiang 150000, P.R. China
}

Received June 18, 2017; Accepted October 3, 2017

DOI: $10.3892 / \mathrm{mmr} .2017 .8031$

\begin{abstract}
Tongue cancer remains a difficult disease to overcome. Long noncoding RNAs (LncRNAs) have been shown to serve significant roles in the diagnosis and treatment of tongue cancer. Herein, the present study aimed to investigate the role of a newly-discovered Lnc, Lnc-EGFR in tongue cancer. The results showed that the transcript level of Lnc-EGFR was upregulated in patients with tongue cancer and in cultured tongue cancer cell lines. Consistently, expression of EGFR was also elevated selectively in cancerous tissues and malignant cell lines. Knockdown of Lnc-EGFR inhibited the clonogenic ability and cell viability of human tongue cancer cell lines UM1 and CAL-27, as evidenced by colony formation assays, and cell proliferation assays. Furthermore, depletion of Lnc-EGFR in UM1 and CAL-27 cells increased cell apoptosis by upregulating the activities of caspase-3, and caspase-9, but not caspase-8. Lnc-EGFR knockdown-mediated inhibition of clonogenic ability and cell viability was rescued by overexpression of EGFR by adding EGFR recombinant protein into both cell lines. Likewise, Lnc-EGFR knockdown-induced cell apoptosis was reversed by co-treatment with recombinant EGFR protein in UM1 and CAL-27 cells. All of these results suggested the oncogenic potential of Lnc-EGFR, which was achieved by positive regulation of EGFR in human tongue cancer.
\end{abstract}

\section{Introduction}

Although great efforts have been made to improve the diagnosis and treatment of tongue cancer, it remains a difficult disease to

Correspondence to: Dr Miaomiao Zhang, Department of Orthodontics, The First Affiliated Hospital of Harbin Medical University, 23 Youzheng Road, Harbin City, Heilongjiang 150000, P.R. China

E-mail: mmzhang1234@yeah.net

Key words: Lnc-EGFR, EGFR, tongue cancer, proliferation, apoptosis cure with a five-year-survival rate of 50\% (1). Multiple therapeutics including surgery, radiotherapy and chemotherapy were applied to advanced tongue cancer in clinic; nevertheless, little improvements were achieved in the past few decades (2). Tongue cancer is more common in older people, but its incidence rate is higher than that of other head and neck cancers even in young people, greatly due to its various risk factors, such as certain environment factors, alcohol intake and even genetic factors (3).

With the rapid development of genome sequencing technologies, the classic view of the transcriptome landscape has undergone a fundamental change (4). It was now well established that more than $90 \%$ of the genome can be transcribed with only less than $2 \%$ being subsequently translated, which means that the vast majority of genome serves as the template for the transcription of noncoding RNAs (ncRNAs) $(5,6)$. Long noncoding RNAs (lncRNAs) are a newly emerged class of noncoding RNA containing more than 200 nucleotides that are widely transcribed in the genome (7). Unlike other noncoding RNAs, lncRNAs involvement in human diseases is largely unclear. Current evidence has implicated that lncRNAs may widely participate in multiple intracellular and extracellular activities, including gene transcription, mRNA splice and tumorigenesis (8). Multiple lncRNAs have been shown to play significant roles in regulating the process of human tongue cancer. For instance, the lncRNA MALAT1 was found to interact with miR-124 and modulate cell growth in human tongue cancer (9). LncRNA HOTTIP was upregulated in human tongue squamous cell carcinoma and its expression correlated with tumor sizing and distant metastasis (10).

The epidermal growth factor receptor (EGFR) is a transmembrane protein, which is a receptor for members of the epidermal growth factor family (EGF family) of extracellular protein ligands $(11,12)$. Mutations that lead to EGFR overexpression or overactivity have been reported in multiple cancers, including squamous-cell carcinoma of lung (more than $80 \%$ cases) (13), anal cancers (14) and epithelial tumors of the head and neck (80-100\% cases) (15). Particularly, EGFR played a prognostic role in the prognosis of tongue cancer (16). Therefore, it is a high priority to uncover the upstream signaling pathway of EGFR and to identify ways to decrease the expression of EGFR from the original source. 
In the present study, we investigated the role of a newly-discovered LncRNA, Lnc-EGFR in human tongue cancer. To this end, a total of 50 tongue cancer patients and four tongue cancer cell lines were used. Cell proliferation and cell apoptosis were detected to examine effects of Lnc-EGFR on tongue cancer proliferation and apoptosis, respectively. Tongue cancer cell lines UM1 and CAL-27 were transfected with specific shRNAs targeting Lnc-EGFR (shLnc-EGFR) with or without the presence of recombinant EGFR protein. Our study is the first to uncover the role of Lnc-EGFR in tongue cancer. Our data might provide novel clues for the diagnosis and treatment of tongue cancer patients in clinic.

\section{Materials and methods}

Human tissues. A total of 50 tongue cancer tissues from patients who were admitted to the Department of Orthodontics, the First Affiliated Hospital of Harbin Medical University between May 2015 and May 2016 (age range, 30-70 years; mean age, 55 years; male: female $=31: 19$ ) were obtained via surgical resection. Their adjacent non-cancerous tissues were also dissected from each patient. All tissues were frozen into liquid nitrogen immediately after dissection and then stored at $-80^{\circ} \mathrm{C}$ till use. All patients showed their full intention to participate in the study and written consent forms were obtained from each patient. The present study was approved by the ethics committee of The First Affiliated Hospital of Harbin Medical University.

Cell culture and shRNAs transfection. Control cells CRL-7421 and tongue cancer cell line SCC-25 were commercially purchased from American Type Tissue Collection (ATCC, Massachusetts, USA). Tongue cancer cell lines HSC-3, UM1 and CAL-27 were purchased from the Cell Bank of Chinese Academy of Sciences (Shanghai, China). All of the cell lines were cultured in dulbecco's modified eagle medium (DMEM; Gibco, Grand Island, NY, USA) supplied with $10 \%$ fetal bovine serum (FBS; Gibco). The cells grew in a $37^{\circ} \mathrm{C}$ incubator with $5 \% \mathrm{CO}_{2}$ and the culture medium was replaced every other day unless otherwise stated. The shRNAs against Lnc-EGFR were synthesized by Genepharm. Co., (Shanghai, China) and the sequences were listed in Table I. The recombinant human EGFR protein was purchased from Abcam Co., (ab155639; NY, USA) and used in a final concentration of $10 \mu \mathrm{M}$. The transfections were performed using Lipofectamine 2000 (Invitrogen, NY, USA) according to the manufactures' instructions in a dose of $2.5 \mu \mathrm{l}$ for $1.5 \mu \mathrm{g}$ DNA. Six $\mathrm{h}$ after transfection, the culture medium was replaced with fresh DMEM supplemented with 10\% FBS.

RNA isolation and RT-PCR. Total RNAs from clinical tissues and cultured cells were extracted with TriZol ${ }^{\circledR}$ reagent (Takara Bio, Inc., Otsu, Japan) in a dilution of $0.5 \mathrm{ml}$ for each well in a 12-well plate. The RNA quality and quantity were determined by Nanodrop 2000 (ThermoFisher Scientific, Inc., NY, USA). Reverse transcription (RT) of first-strand cDNAs was performed with PrimeScript RT Master Mix (Perfect Real Time; Takara) following the manufacturer's protocol. All PCR reactions were performed in an ABI PRISM 7900 Real-Time system (Thermo Fisher Scientific, Inc.) with the SYBR ${ }^{\circledR}$ Premix Ex Taq ${ }^{\mathrm{TM}}$ kit (Takara Bio, Inc.). The thermocycling
Table I. Sequences of the primers used in reverse transcription-quantitative polymerase chain reaction and the sequences of shRNAs against Lnc-EGFR.

\begin{tabular}{ll}
\hline Gene & \multicolumn{1}{c}{ Primer nucleotide sequences } \\
\hline Lnc-EGFR & \\
Forward & 5'-CAGCAGCCCTGCAATTAAAC-3' \\
Reverse & 5'-GGGTCCTCATGTAATGGTAATAGG-3' \\
EGFR & \\
Forward & 5'-AGGCACGAGTAACAAGCTCAC-3' \\
Reverse & 5'-ATGAGGACATAACCAGCCACC-3' \\
GAPDH & \\
Forward & 5'-GTGGACATCCGCAAAGAC-3' \\
Reverse & 5'-AAAGGGTGTAACGCAACTA-3' \\
shLnc-EGFR-1 & 5'-GCTCTGCTTTAGTCAGGGT-3' \\
shLnc-EGFR-2 & 5'-TACATGCCATCCTGGCCAT-3' \\
\hline
\end{tabular}

ShRNA, short hairpin RNA; Lnc, long coding RNA; EGFR, epidermal growth factor receptor.

protocol was shown as follows: Initial denaturation at $95^{\circ} \mathrm{C}$ for $2 \mathrm{~min}$, followed by 35 repeats of the three-step cycling program consisting of $30 \mathrm{sec}$ at $95^{\circ} \mathrm{C}$ (denaturation), $1 \mathrm{~min}$ at $53^{\circ} \mathrm{C}$ (primer annealing) and $30 \mathrm{sec}$ at $72^{\circ} \mathrm{C}$ (elongation), followed by a final extension step for $10 \mathrm{~min}$ at $72^{\circ} \mathrm{C}$. The housekeeping gene GAPDH was included as an internal control. Primer sequences were listed in Table I. All quantitative data were normalized to GAPDH using the $2^{-\Delta \Delta \mathrm{Ct}}$ method (17).

Western blot analysis. Briefly, total proteins from human tissues and cells were collected by lysis buffer (RIPA, Beyotime, Nantong, China) on ice and quantified using Bio-Rad protein assay reagent (Beyotime). Equal amounts of protein $(40 \mu \mathrm{g})$ were loaded onto $12 \%$ sodium dodecyl sulfate-polyacrylamide gel (SDS-PAGE) and transferred to a $0.22 \mu \mathrm{m}$ nitrocellulose membrane (NC, Millipore, MA, USA). The membrane was blocked for $1 \mathrm{~h}$ with $5 \%$ skimmed milk at room temperature and then incubated with primary antibodies overnight at $4^{\circ} \mathrm{C}$. The primary antibodies against EGFR (SAB5500096, 1:1,000) was purchased from Sigma Co. (NY, USA) and the primary antibody against Tublin was from Santa Cruz Biotech (Santa Cruz, CA, USA). After washing with TBST for 4 times (8 min each time), the membrane was then incubated with secondary goat anti-rabbit antibody (sc-2004; Santa Cruz Biotech) for $1 \mathrm{~h}$ at $37^{\circ} \mathrm{C}$ with a dilution of 1:1,000. Finally, the proteins were quantified using ECL Prime Western Blotting Detection reagent (GE Healthcare, Parsippany, NJ, USA) and an ImageQuant LAS 4000 Mini Biomolecular Imager (GE Healthcare).

Colony formation assay. UM1 and CAL-27 cells were transfected with shLnc-EGFR or control shRNAs (shNC) with or without the presence of EGFR recombinant protein in six-well plates with a density of 200 cells/well, during which the culture medium was not changed. After 2 weeks in $37^{\circ} \mathrm{C}$ incubator, the cell colonies that contained more than 50 cells 

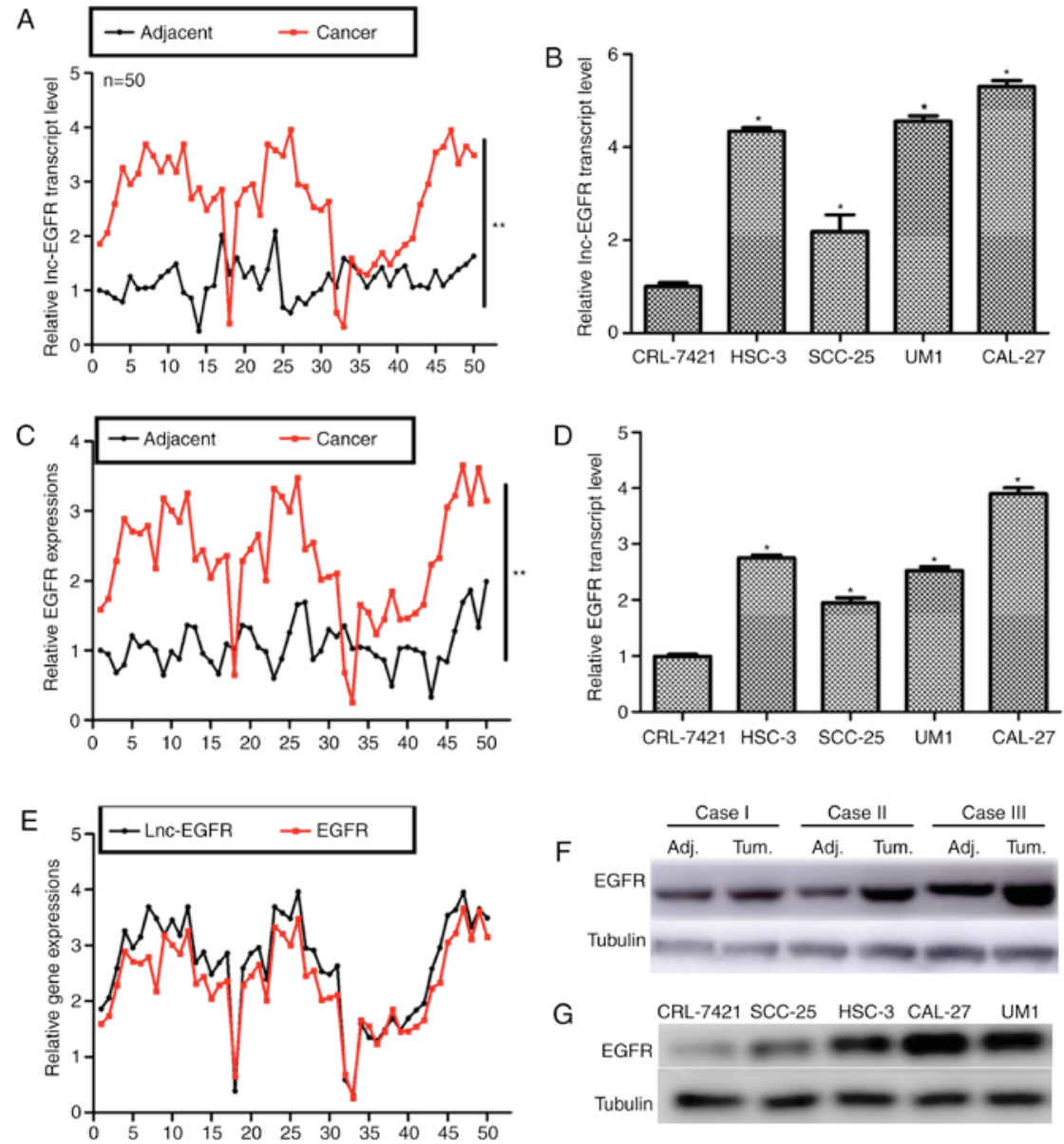

Figure 1. Expression of EGFR and long noncoding RNA Lnc-EGFR were upregulated in human tongue cancer. (A) RT-PCR analysis of the transcript level of Lnc-EGFR in 50 tongue cancer patients. "* $\mathrm{P}<0.01$, Cancer vs. Adjacent. (B) RT-PCR analysis of the relative transcript level of Lnc-EGFR in four tongue cancer cell lines as compared with control CRL-7421 cells. "P<0.05, vs. CRL-7421 cells. (C) RT-PCR analysis of the transcript level of EGFR in 50 tongue cancer patients. ${ }^{* * *} \mathrm{P}<0.01$, Cancer vs. Adjacent. (D) RT-PCR analysis of the relative transcript level of EGFR in four tongue cancer cell lines as compared with control CRL-7421 cells. "P<0.05, vs. CRL-7421 cells. (E) The association of Lnc-EGFR and EGFR was assessed in clinical tissues with RT-PCR. (F) Protein levels of EGFR in 3 randomly selected tongue cancer tissues by western blot analysis. (G) Protein levels of EGFR in four tongue cancer cell lines and in control CRL-7421 cells by western blot analysis. Tubulin was synchronously blotted as a loading control.

were counted by staining with crystal violet $(0.5 \%)$ for $10 \mathrm{~min}$ at room temperature and observed under a light microscope with a magnification of 200 (Nikon, Japan).

Cell proliferation assay. Both UM1 and CAL-27 cells were seeded in a 96-well plate at a concentration of 1,000 cells/well. After incubation for $24 \mathrm{~h}$, cells were transfected with shLnc-EGFR or control shRNAs (shNC) with or without the presence of EGFR recombinant protein. Cell viability was monitored in a consecutive 5 days with a CellTiter 96 AQueous Non-Radioactive Cell Proliferation kit (Promega Corporation, Madison, WI, USA) as per the manufacturer's protocols. The cell viability was determined by collecting the absorbance at $490 \mathrm{~nm}$ using a microplate reader (Tecan, Männedorf, Switzerland).

Flow cytometric analysis of cell apoptosis. The annexin V/PI assay was performed as per the manufacturer's instructions (Invitrogen). Briefly, UM1 and CAL-27 cells were plated into 6-well plates and transfected with shLnc-EGFR or control shRNAs (shNC) with or without the presence of EGFR recombinant protein for $48 \mathrm{~h}$. Afterwards, cells were washed with pre-cold PBS, trypsinized and re-suspended in $100 \mu \mathrm{l}$ of binding buffer with $2.5 \mu 1$ FITC conjugated annexin-v and $1 \mu \mathrm{l}$ PI $(100 \mu \mathrm{g} / \mathrm{ml})$. Afterwards, cells were incubated at room temperature for $15 \mathrm{~min}$ in darkness. A total of at least 10,000 cells were collected and calculated by flow cytometry for both cell lines (BD Biosciences, San Diego, CA, USA).

Determination of caspase activities. The activities of caspase-3, caspase- 8 and caspase- 9 were determined by the caspase activity kits (Beyotime) based on the instructions. Briefly, cells were transfected with shRNAs for $48 \mathrm{~h}$. Afterwards, cell lysates were collected by low speed centrifuge $(1,000 \mathrm{~g}, 5 \mathrm{~min}$, $4^{\circ} \mathrm{C}$ ). An equal amount of $10 \mu \mathrm{l}$ proteins from each sample was added into 96-well plates and mixed with an aliquot of $80 \mu \mathrm{l}$ reaction buffer supplied with caspase substrates $(2 \mathrm{mM})$. After incubated at $37^{\circ} \mathrm{C}$ for $4 \mathrm{~h}$, caspase activities were determined by the TECAN reader at an absorbance wavelength of $450 \mathrm{~nm}$.

Immunofluorescence staining. Briefly, cells were cultured on a coverslip in six-well plates at a density of 10,000 cells/well and then fixed with cold acetone on ice for $20 \mathrm{~min}$. After washed with PBS, cells were blocked with normal goat serum 
Table II. Association of Lnc-EGFR with clinical variables among 50 tongue cancer patients.

\begin{tabular}{|c|c|c|c|c|}
\hline \multirow[b]{2}{*}{ Variable } & \multirow[b]{2}{*}{ Numbers } & \multicolumn{2}{|c|}{ Expression of Lnc-EGFR } & \multirow[b]{2}{*}{ P-value } \\
\hline & & Low $(n=34)$ & High $(n=16)$ & \\
\hline Age (year) & & & & 0.47 \\
\hline$<40$ & 8 & 7 & 1 & \\
\hline $40-50$ & 16 & 10 & 6 & \\
\hline$>50$ & 26 & 17 & 9 & \\
\hline Sex & & & & 0.228 \\
\hline Male & 31 & 19 & 12 & \\
\hline Female & 19 & 15 & 4 & \\
\hline Tumor size $(\mathrm{T})$ & & & & $<0.001^{\mathrm{a}}$ \\
\hline $\mathrm{T} 1$ and $\mathrm{T} 2(\leq 4 \mathrm{~cm})$ & 39 & 32 & 7 & \\
\hline $\mathrm{T} 3$ and $\mathrm{T} 4$ ( $>4 \mathrm{~cm}$ or any size with distant metastasis) & 11 & 2 & 9 & \\
\hline Lymph node metastasis $(\mathrm{N})$ & & & & 0.191 \\
\hline No & 35 & 26 & 9 & \\
\hline N1 or above & 15 & 8 & 7 & \\
\hline Distant metastasis (M) & & & & 0.486 \\
\hline M0 & 38 & 27 & 11 & \\
\hline M1 & 12 & 7 & 5 & \\
\hline TNM stage & & & & 0.103 \\
\hline $\mathrm{I} / \mathrm{II}$ & 34 & 26 & 8 & \\
\hline III/IV & 16 & 8 & 8 & \\
\hline
\end{tabular}

TNM, tumor node metastasis; Lnc, long coding RNA; EGFR, epidermal growth factor receptor.

for 10 min supplied with $0.1 \%$ Triton $\mathrm{X}-10$ and then incubated with primary antibody against EGFR $(1: 250)$ at $37^{\circ} \mathrm{C}$ for $1 \mathrm{~h}$ and then overnight at $4^{\circ} \mathrm{C}$. Secondary antibodies (Dylight 549) were purchased from Dylight (Abcam) and used in a dilution of $1: 1,000$ at $37^{\circ} \mathrm{C}$ for $1 \mathrm{~h}$. The photos were taken with a Nikon camera.

Statistical analysis. All experiments were repeated at least three times in triplicate to obtain reproducible results. All data were presented as mean \pm standard deviation (SD). Student's t-test analysis was used for the comparison between two groups with Microsoft Excel 2007. Two-way ANOVA analysis was included for the comparisons among three or more groups. Data were analyzed with GraphPad Prism 6 (GraphPad Software Inc., San Diego, CA, USA). Any value of $\mathrm{P}<0.05$ was considered statistically significant.

\section{Results}

Expression of EGFR and long noncoding RNA Lnc-EGFR were upregulated in human tongue cancer. In the present study, a total of 50 tongue cancer patients were involved and the transcript level of Lnc-EGFR was assessed. As shown in Fig. 1A, only 3 of the 50 tongue cancer patients showed lower expression of Lnc-EGFR as compared with the adjacent non-cancerous tissues $(\mathrm{P}<0.01)$. Next, four tongue cancer cell lines were cultured and subjected for RT-PCR analysis. All of the four tongue cancer cell lines exhibited higher transcript levels of Lnc-EGFR as compared with the control CRL-7421 cell. Particularly, UM1 and CAL-27 showed the highest expressions of Lnc-EGFR (Fig. 1B). Thus, these two cell lines were selected for subsequent functional analysis. Clinical characteristics were also analyzed in Table II. The patients were divided into two categories based on the expression of Lnc-EGFR: high level of Lnc-EGFR $(n=16)$ denotes those with transcript levels of Lnc-EGFR higher than its median and low level of Lnc-EGFR $(n=34)$ denotes those with transcript level of Lnc-EGFR lower than the median level. Among the examined variables (age, sex, tumor size, lymph node metastasis, distant metastasis and TNM stage), the expression of Lnc-EGFR was only associated with tongue tumor size $(\mathrm{P}<0.001)$. Therefore, the effects of Lnc-EGFR on cell proliferation and apoptosis were thereafter explored.

Expression of EGFR was also detected with RT-PCR and western blot analysis, since Lnc-EGFR was predicted as a potential enhancer of EGFR (18). It was shown in Fig. 1C and $\mathrm{D}$, the transcription levels of EGFR were significantly increased in both clinical tongue cancer tissues and in cultured tongue cancer cells. The association of Lnc-EGFR and EGFR was also analyzed in clinical tissues (Fig. 1E). Furthermore, the protein level of EGFR was also upregulated in 3 randomly selected tongue cancer patients as depicted in Fig. 1F. Total proteins were also extracted from cultured cells and it was shown that the expression of EGFR was remarkably higher in all of the four tongue cancer cell lines (Fig. 1G). All of these results suggested that in parallel to EGFR, Lnc-EGFR was notably increased in human tongue cancer. 

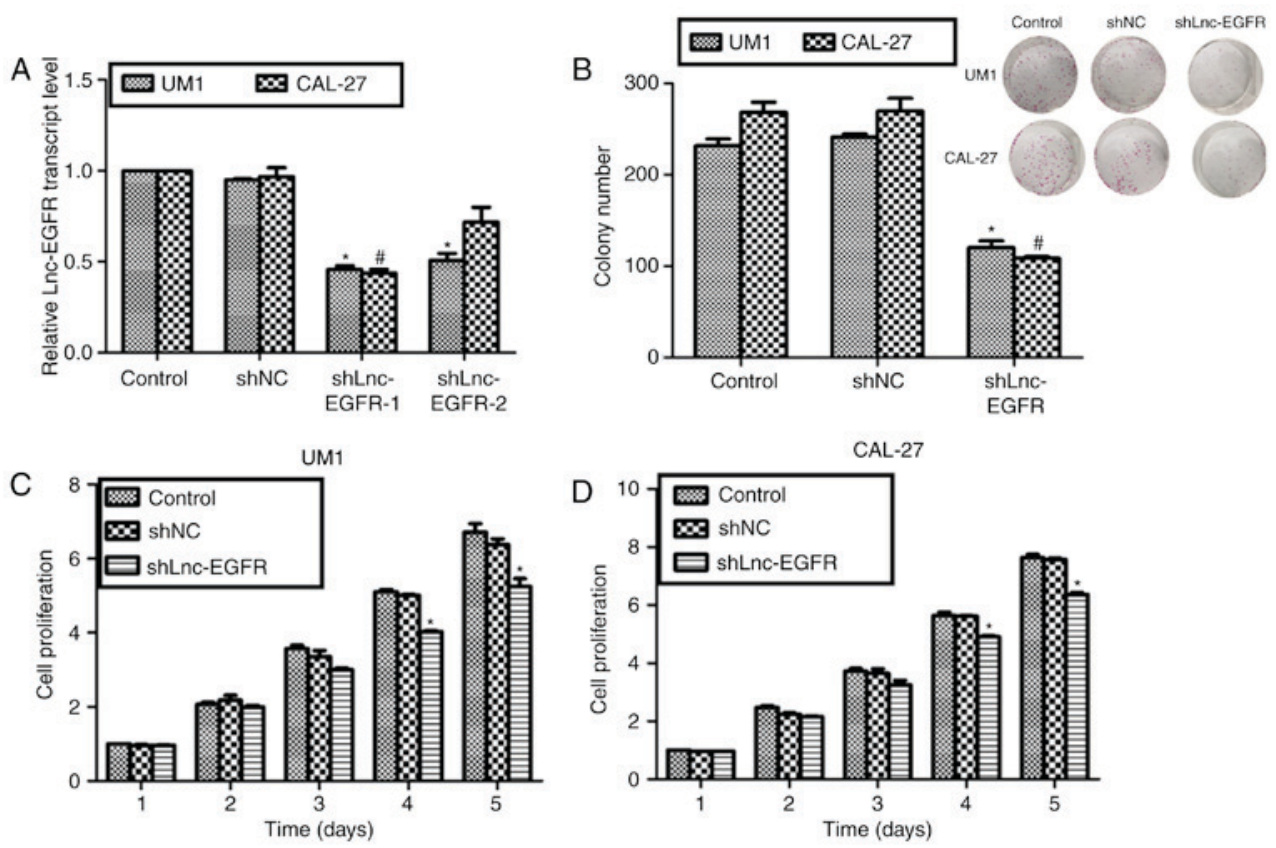

Figure 2. Knockdown of Lnc-EGFR inhibited cell proliferation in human tongue cancer cells. (A) Two specific shRNAs against Lnc-EGFR were transfected into UM1 and CAL-27 cells and RT-PCR analysis was performed to assess the expression of Lnc-EGFR. (B) Colony formation assays were performed in UM1 and CAL-27 cells upon transfection of shLnc-EGFR. The right upper panels were representative images of colony formation assay. "P<0.05, vs. Control in UM1 cells. " $\mathrm{P}<0.05$, vs. Control in CAL-27 cells. (C) Cell proliferation assays were performed in a consecutive 5 days when UM1 cells were transfected with shLnc-EGFR. (D) Cell proliferation assays were performed in a consecutive 5 days when CAL-27 cells were transfected with shLnc-EGFR. ${ }^{*} \mathrm{P}<0.05$, vs. Control.
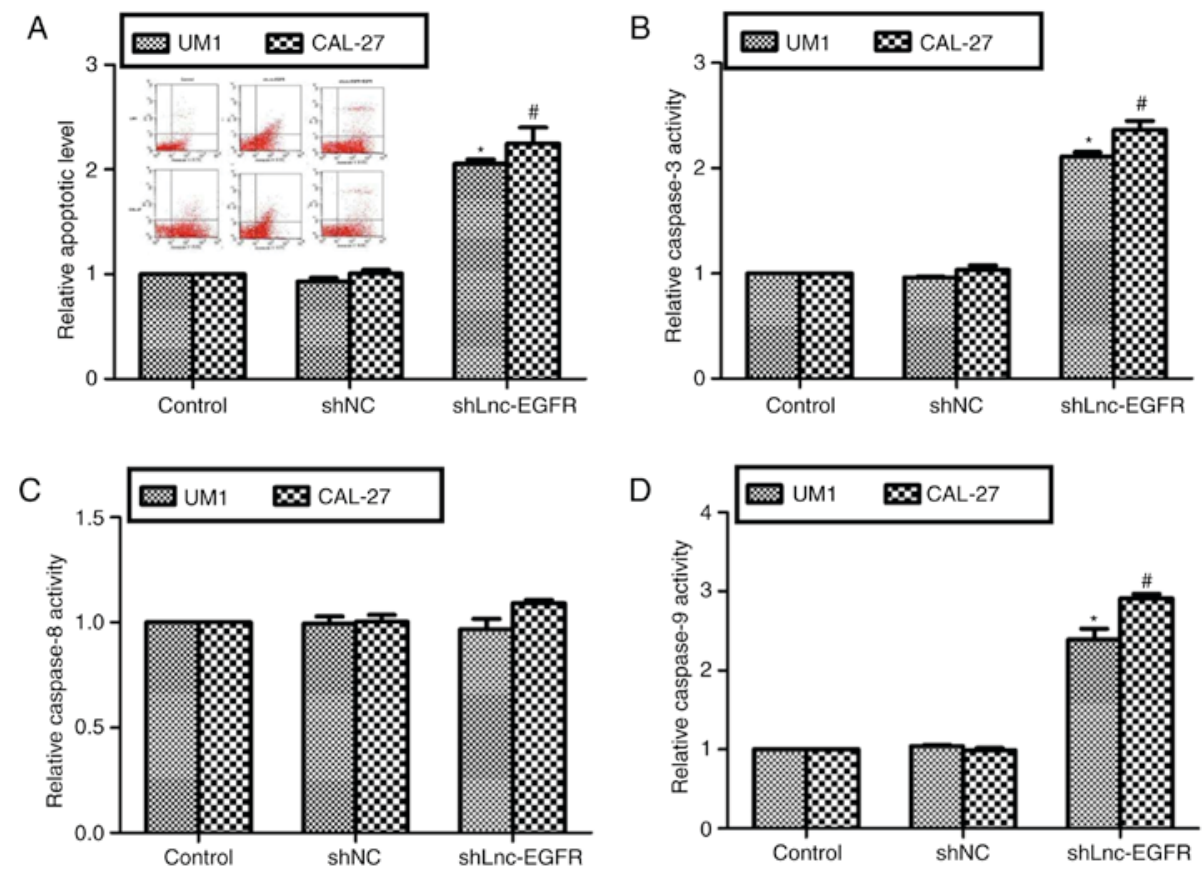

Figure 3. Knockdown of Lnc-EGFR increased cell apoptosis in human tongue cancer in vitro. (A) Cell apoptosis was assessed in UM1 and CAL-27 cells upon knockdown of Lnc-EGFR. The left upper panels were representative images of cell apoptosis assays. (B) The relative activity of caspase-3 was examined in UM1 and CAL-27 cells upon transfection of shLnc-EGFR. (C) The relative activity of caspase-8 was examined in UM1 and CAL-27 cells upon transfection of shLnc-EGFR. (D) The relative activity of caspase-9 was examined in UM1 and CAL-27 cells upon transfection of shLnc-EGFR. "P<0.05, vs. Control in UM1 cells. ${ }^{*} \mathrm{P}<0.05$, vs. Control in CAL-27 cells.

Knockdown of Lnc-EGFR inhibited cell proliferation in human tongue cancer cells. To explore the roles of Lnc-EGFR, two specific shRNAs against Lnc-EGR were synthesized and transfected into UM1 and CAL-27 cells. The expression of
Lnc-EGFR was decreased by more than $50 \%$ in both cell lines upon shLnc-EGFR-1 transfection; however, shLnc-EGFR-2 was only effective for UM1 cells (Fig. 2A). Thus, shLnc-EGFR-1 was selected and renamed as shLnc-EGFR. Colony formation 

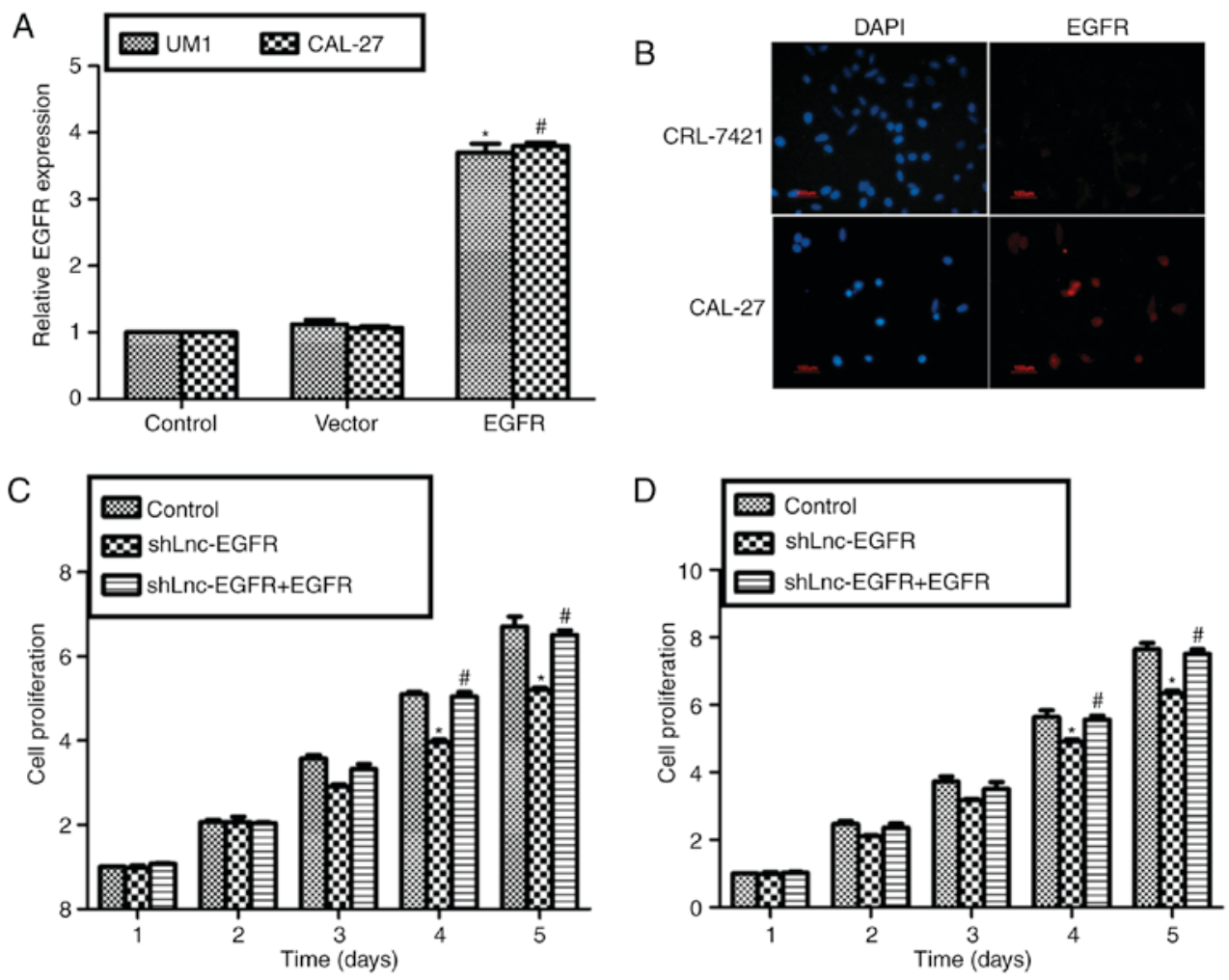

Figure 4. Lnc-EGFR promoted cell proliferation through EGFR in human tongue cancer cells. (A) The expression of EGFR was examined with RT-PCR when UM1 and CAL-27 cells were treated with a recombinant EGFR protein. (B) Immunofluorescence assays were performed in CRL-7421 and CAL-27 cells, showing the expression of EGFR in cultured cells. (C) Cell proliferation assays were performed in UM1 cells when cells were transfected with shLnc-EGFR with or without the presence of recombinant EGFR protein. (D) Cell proliferation assays were performed in CAL-27 cells when cells were transfected with shLnc-EGFR with or without the presence of recombinant EGFR protein. ${ }^{*} \mathrm{P}<0.05$, vs. Control in UM1 cells. "P<0.05, vs. Control in CAL-27 cells.

assays and cell proliferation assays were performed to reveal the roles of Lnc-EGFR. Approximate 230 colonies and 275 colonies were observed in control UM1 cells and CAL-27 cells, respectively; however, only an average of 110 colonies in UM1 cells and 105 colonies in CAL-27 cells were counted upon shLnc-EGFR transfection, while control shRNA caused no effects on both cell lines (Fig. 2B). As for the cell proliferation assays, there were no notable difference among the three experimental groups in the former three days for both UM1 and CAL-27 cells. Interestingly, the proliferative rate of UM1 was suppressed by $24 \%$ on the fourth day and $29 \%$ on the fifth day (Fig. 2C). Likewise, knockdown of Lnc-EGFR with shLnc-EGFR inhibited cell proliferative rate on the fourth and fifth day in CAL-27 cells (Fig. 2D). These data suggested that knockdown of Lnc-EGFR in human tongue cancer suppressed cell proliferation in vitro.

Knockdown of Lnc-EGFR increased cell apoptosis in human tongue cancer in vitro. Next, cell apoptotic rates were assessed in UM1 and CAL-27 cells. As shown in Fig. 3A, upon knockdown of Lnc-EGFR for $72 \mathrm{~h}$, the apoptotic rate was increased to 2-fold in UM1 cells and 2.2-fold in CAL-27 cells. Cell apoptosis have two classical signal pathways: intrinsic pathway (caspase-3 and caspase-9) and extrinsic pathway (capsase-8) (19). It was shown in Fig. 3B, the relative caspase-3 activities were remarkably increased in both cell lines when Lnc-EGFR was knocked down with shLnc-EGFR. However, the relative caspase- 8 activities remained unchanged upon transfection of shLnc-EGFR (Fig. 3C). Meanwhile, the activities of caspase-9 were increased by more than 1-fold in both cell lines (Fig. 3D). All of above observations suggested that Lnc-EGFR suppressed cell apoptosis through intrinsic pathway in human tongue cancer in vitro.

Lnc-EGFR promoted cell proliferation through EGFR in human tongue cancer cells. Both UM1 and CAL-27 cells showed elevated expression of EGFR when recombinant protein EGFR were added into both cells lines (Fig. 4A). Immunofluorescence staining showed that there was almost no EGFR expression in the control cell line CRL-7421 and the expression of EGFR was obviously upregulated in CAL-27 cells (Fig. 4B). Similarly, although cell proliferative rate was decreased upon transfection with shLnc-EGFR in UM1 cells (Fig. 4C) and CAL-27 cells (Fig. 4D), the cell proliferative capacity was recovered to basic level when shLnc-EGFR and recombinant EGFR protein co-treated each cell line (Fig. 4C and D). Altogether with Fig. 2, our findings indicated Lnc-EGFR increased cell proliferation through EGFR in human tongue cancer cell lines UM1 and CAL-27.

Lnc-EGFR suppressed cell apoptosis through EGFR in human tongue cancer cells. We also examined the effects of EGFR re-expression on Lnc-EGFR knockdown-mediated cell apoptosis. As shown in Fig. 5, transfection of shLnc-EGFR increased cell apoptosis to 2-fold in both cell lines, while the cell apoptotic rate was decreased to the normal level when cells were co-treated with shLnc-EGFR and recombinant EGFR protein. Similarly, the relative activities of caspase-3 (Fig. 5B) 
A

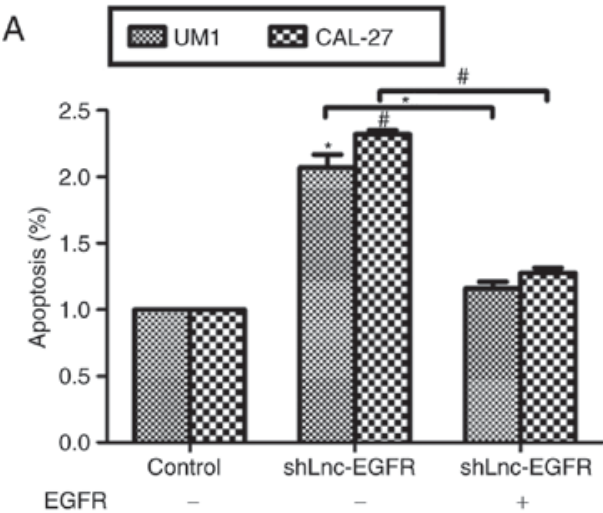

C

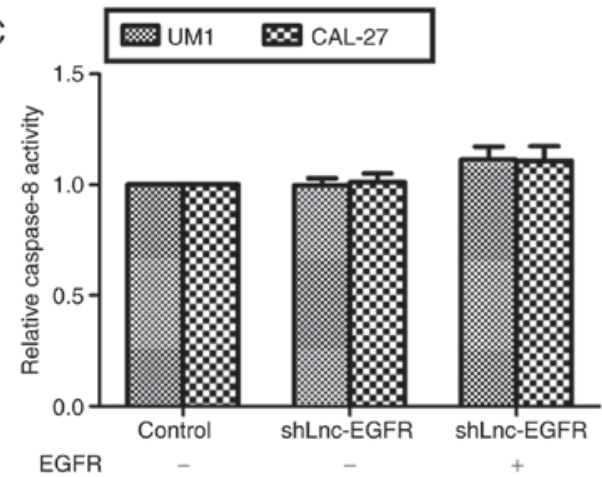

B

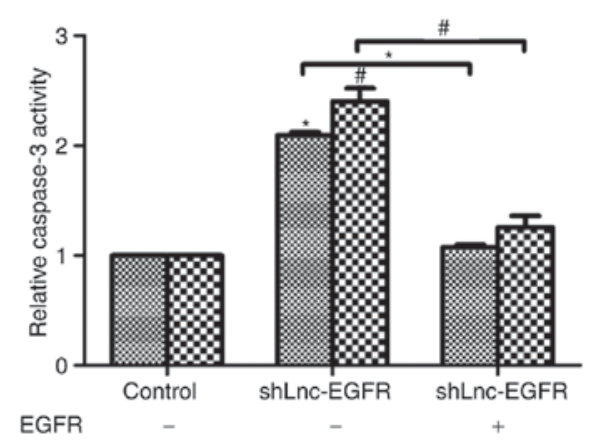

D

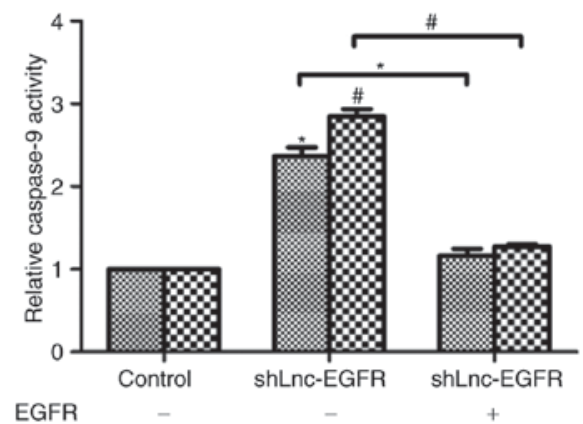

Figure 5. Lnc-EGFR suppressed cell apoptosis through EGFR in human tongue cancer cells. (A) Cell apoptosis were examined in CAL-27 and CAL-27 cells when cells were transfected with shLnc-EGFR with or without the presence of recombinant EGFR protein. (B) The relative activities of caspase-3 were detected in CAL-27 and CAL-27 cells when cells were transfected with shLnc-EGFR with or without the presence of recombinant EGFR protein. (C) The relative activities of caspase-8 were examined in CAL-27 and CAL-27 cells when cells were transfected with shLnc-EGFR with or without the presence of recombinant EGFR protein. (D) The relative activities of caspase-9 were examined in CAL-27 and CAL-27 cells when cells were transfected with shLnc-EGFR with or without the presence of recombinant EGFR protein. "P<0.05, vs. Control in UM1 cells. "P<0.05, vs. Control in CAL-27 cells.

and caspase-9 (Fig. 5D) were increased by knockdown of Lnc-EGFR and recovered by co-treated with EGFR recombinant protein in both UM1 and CAL-27 cells. However, the caspase- 8 activity remained unchanged despite any treatment (Fig. 5C). These data suggested that Lnc-EGFR suppressed cell apoptosis through positive regulation of EGFR in human tongue cancer cells in vitro.

\section{Discussion}

This is a preliminary study on the role of Lnc-EGFR in human tongue cancer. In this study, we demonstrated the relative transcript level of Lnc-EGFR was upregulated in clinical tongue cancer tissues and in cultured tongue cancer cells, which was consistent with that of EGFR. Knockdown of Lnc-EGFR inhibited colony formation and cell proliferative rates in tongue cancer cells UM1 and CAL-27, and increased cell apoptosis by enhancing the activities of caspase- 3 and caspase-9, but not caspase-8. More interestingly, re-expression of EGFR in Lnc-EGFR-depleted UM1 and CAL-27 cells rescued Lnc-EGFR depletion-mediated inhibition of cell proliferative ability and promotion of cell apoptotic capacity. These results suggested that the oncogenic potential of Lnc-EGFR was achieved by upregulating the expression of EGFR.

Lnc-EGFR is a newly identified long noncoding RNA using high-throughput screening in Treg cells in hepatocellular carcinoma (18). Lnc-EGFR upregulation in Tregs correlates positively with the tumor size and expression of EGFR/Foxp3, but negatively with IFN-g expression in patients and xenografted mouse models. Lnc-EGFR stimulates Treg differentiation, suppresses CTL activity and promotes HCC growth in an EGFR-dependent manner (18). Consistent with this pioneer study, we also showed that Lnc-EGFR was significantly increased in tongue cancer tissues. Altogether, the results by others and us might suggested that Lnc-EGFR is a novel oncogene gene. Lnc-EGFR might play a wide range of critical roles in solid tumors. However, it remains scarce that how Lnc-EGFR functions in solid tumors. We provided evidence that Lnc-EGFR positively regulated the expression of EGFR, an important member of the receptor tyrosine kinase family. Previously, it has been indicated that Lnc-EGFR specifically binds to EGFR and blocks its interaction with and ubiquitination by c-CBL, stabilizing it and augmenting activation of itself and its downstream AP-1/NF-AT1 axis, which in turn elicits EGFR expression (18). Hence, we speculated that Lnc-EGFR might directly bind to EGFR and regulate its expression in human tongue cancer. However, it merits further investigation of this detailed interaction and other possible mechanisms underlying Lnc-EGFR functions remains to be further elucidated in tongue cancer.

The induction of apoptosis is a good basis for anticancer treatment and a valuable guide to predict tumor response after 
anticancer therapies are monitored (20). Two major pathways are well-known to be involved in the initiation of apoptosis: the mitochondria-induced intrinsic pathway and the death receptor-mediated extrinsic pathway (21). In the intrinsic pathway, once cytochrome $\mathrm{c}$ is released, it binds with apoptotic protease activating factor-1 (Apaf-1) and ATP, which then bind to pro-caspase- 9 to create a protein complex known as an apoptosome. The apoptosome cleaves the pro-caspase to its active form of caspase-9, which in turn activates the effector caspase-3 (22). In the extrinsic pathway, the Fas (first apoptosis signal) receptor binds to the Fas ligand (FasL) and results in the formation of the death-inducing signaling complex (DISC), which contains the FADD, caspase-8 and caspase-10 (23). Therefore, we examined the relative activities of caspase-3, caspase- 8 and caspase- 9 and demonstrated that only intrinsic pathway was involved in Lnc-EGFR-regulated cell apoptosis in human tongue cancer.

In total, our study was the first one to identify the role of Lnc-EGFR in human tongue cancer and suggested that Lnc-EGFR functioned by positive regulation of EGFR. Lnc-EGFR was shown to promote cell proliferation and inhibit cell apoptosis in vitro and this phenomenon could be reversed by decreasing EGFR. Due to the key role of EGFR in tumorigenesis, this study suggested the diagnostic value of Lnc-EGFR for tongue cancer and might provide novel insights into the development of therapeutic strategies for treatment of tongue cancer.

\section{References}

1. Leemans CR, Braakhuis BJ and Brakenhoff RH: The molecular biology of head and neck cancer. Nat Rev Cancer 11: 9-22, 2011.

2. Hasegawa H, Kusumi Y, Asakawa T, Maeda M, Oinuma T, Furusaka T, Oshima T and Esumi M: Expression of von hippel-lindau tumor suppressor protein (pVHL) characteristic of tongue cancer and proliferative lesions in tongue epithelium. BMC Cancer 17: 381, 2017.

3. Scully C, Field JK and Tanzawa H: Genetic aberrations in oral or head and neck squamous cell carcinoma 3: Clinico-pathological applications. Oral Oncol 36: 404-413, 2000.

4. de Hoon M, Shin JW and Carninci P: Paradigm shifts in genomics through the FANTOM projects. Mamm Genome 26: 391-402, 2015

5. Xin Y, Li Z, Shen J, Chan MT and Wu WK: CCAT1: A pivotal oncogenic long non-coding RNA in human cancers. Cell Prolif 49: 255-260, 2016

6. Guo X and Hua Y: CCAT1: An oncogenic long noncoding RNA in human cancers. J Cancer Res Clin Oncol 143: 555-562, 2017.

7. Yang YT, Wang YF, Lai JY, Shen SY, Wang F, Kong J, Zhang W and Yang HY: Long non-coding RNA UCA1 contributes to the progression of oral squamous cell carcinoma by regulating the WNT/B-catenin signaling pathway. Cancer Sci 107: 1581-1589, 2016

8. Wang L, Ye S, Wang J, Gu Z, Zhang Y, Zhang C and Ma X: HuR stabilizes lnc-Sox 5 mRNA to promote tongue carcinogenesis. Biochemistry (Mosc) 82: 438-445, 2017.
9. Zhang TH, Liang LZ, Liu XL, Wu JN, Su K, Chen JY, Zheng QY, Huang HZ and Liao GQ: Long non-coding RNA MALAT1 interacts with miR-124 and modulates tongue cancer growth by targeting JAG1. Oncol Rep 37: 2087-2094, 2017.

10. Zhang H, Zhao L, Wang YX, Xi M, Liu SL and Luo LL: Long non-coding RNA HOTTIP is correlated with progression and prognosis in tongue squamous cell carcinoma. Tumour Biol 36: 8805-8809, 2015 .

11. Arteaga CL: Overview of epidermal growth factor receptor biology and its role as a therapeutic target in human neoplasia. Semin Oncol 29 (5 Suppl 14): S3-S9, 2002.

12. Herbst RS: Review of epidermal growth factor receptor biology. Int J Radiat Oncol Biol Phys 59 (2 Suppl): S21-S26, 2004.

13. Gao JW, Zhan P, Qiu XY and Song Y: Erlotinib-based doublet targeted therapy versus erlotinib alone in previously treated advanced non-small-cell lung cancer: A meta-analysis from 24 randomized controlled trials. Oncotarget 32: 2017.

14. Walker F, Abramowitz L, Benabderrahmane D, Duval X, Descatoire V, Hénin D, Lehy T and Aparicio T: Growth factor receptor expression in anal squamous lesions: Modifications associated with oncogenic human papillomavirus and human immunodeficiency virus. Hum Pathol 40: 1517-1527, 2009.

15. Alterio D, Marvaso G, Maffini F, Gandini S, Chiocca S, Ferrari A, Preda L, Rocca MC, Lepanto D, Fodor C, et al: Role of EGFR as prognostic factor in head and neck cancer patients treated with surgery and postoperative radiotherapy: Proposal of a new approach behind the EGFR overexpression. Med Oncol 34: 107, 2017.

16. Lindell Jonsson E, Nylander K, Hallén L and Laurell G: Immunohistochemical analysis of EGFR and hyaluronan in tongue cancer and the development of regional recurrence in patients initially diagnosed N0. Acta Otolaryngol 137: 877-882, 2017.

17. Livak KJ and Schmittgen TD: Analysis of relative gene expression data using real-time quantitative PCR and the 2(-Delta Delta C(T)) method. Methods 25: 402-408, 2001.

18. Jiang R, Tang J, Chen Y, Deng L, Ji J, Xie Y, Wang K, Jia W, Chu WM and Sun B: The long noncoding RNA lnc-EGFR stimulates T-regulatory cells differentiation thus promoting hepatocellular carcinoma immune evasion. Nat Commun 8: 15129, 2017.

19. Islam S, Qi W, Morales C, Cooke L, Spier C, Weterings E and Mahadevan D: Disruption of aneuploidy and senescence induced by aurora inhibition promotes intrinsic apoptosis in double hit or double expressor diffuse large b-cell lymphomas. Mol Cancer Ther: doi: 10.1158/1535-7163, 2017.

20. Li HH, Su JH, Chiu CC, Lin JJ, Yang ZY, Hwang WI, Chen YK, Lo YH and $\mathrm{Wu}$ YJ: Proteomic investigation of the sinulariolide-treated melanoma cells A375: Effects on the cell apoptosis through mitochondrial-related pathway and activation of caspase cascade. Mar Drugs 11: 2625-2642, 2013.

21. Liu JY, Liu Z, Wang DM, Li MM, Wang SX, Wang R, Chen JP, Wang YF and Yang DP: Induction of apoptosis in K562 cells by dicyclohexylammonium salt of hyperforin through a mitochondrial-related pathway. Chem Biol Interact 190: 91-101, 2011.

22. Kerr JF, Wyllie AH and Currie AR: Apoptosis: A basic biological phenomenon with wide-ranging implications in tissue kinetics. Br J Cancer 26: 239-257, 1972.

23. Wajant H: The Fas signaling pathway: More than a paradigm. Science 296: 1635-1636, 2002. 